\title{
Resolution Versus Error for Computational Electron Microscopy
}

\author{
Lorenzo Luzi ${ }^{1}$, Andrew Stevens ${ }^{1,2}$, Hao Yang ${ }^{3}$, and Nigel D. Browning ${ }^{1,4}$ \\ 1. Pacific Northwest National Laboratory, Richland, WA USA \\ 2. Duke University, ECE, Durham, NC USA \\ 3. Lawrence Berkeley National Laboratory, Berkeley, CA USA \\ 4. University of Washington, Materials Science and Engineering, Seattle, WA USA
}

Images that are collected via scanning transmission electron microscopy (STEM) can be undersampled to avoid damage to the specimen while maintaining resolution [1,2]. We have used BPFA to impute missing data and reduce noise [3]. The reconstruction is typically evaluated using the peak signal-to-noise ratio (PSNR). This measure is too conservative for STEM images and we propose that the Fourier ring correlation (FRC) is used instead to evaluate the reconstruction. We are not concerned with exact reconstruction of the truth image, and therefore PSNR is a conservative estimation of the quality of the reconstruction. Instead, we are concerned with the visual resolution of the image and whether atoms can be distinguished. We have evaluated the reconstruction of a simulated STEM image using the FRC and compared the results with the PSNR measurements. The FRC captures the resolution of the image and is not affected by a large MSE if the atom peaks are still distinguishable. The noisy and reconstructed images are shown in Figure 1.

The simulated STEM image was sampled at 100\%, $80 \%$, 40\%, and $20 \%$ of the original pixels to simulate an undersampled scan. The reconstruction used half-overlapping $10 \mathrm{x} 10$ patches. The dictionary size is 64 and 30 iterations were completed during each reconstruction. The 100\% image was denoised instead of reconstructed. Poisson noise was applied to the simulated image with $\lambda$ values of $1000,500,50,5$, and 2.5 to simulate lower imaging dose. The original simulated STEM image was generated using a dose of 1000 .

The simulated STEM image is 100 by 100 pixels and has essentially no high frequency components. The image reconstruction tends to smooth the data, also resulting in no high frequency components. This causes the FRC of the two images to be large at higher resolutions and may be misleading. For this reason, the BPFA has only some overlap to avoid excessive smoothing. Moreover, the resolution of the simulated image is approximately $3.9 \AA^{-1}$, so we only look that far in the frequency domain when performing the FRC. If the FRC curve does not cross over a threshold criteria, a resolution value of 3.9 is used. We emphasize that our reported results are conservative.

The FRC and PSNR values using the ground truth and the reconstructed images are shown in Tables 1 and 2. The left side show the metrics without using BPFA (missing pixels) and the right side show the metrics after using BPFA. When we did not use BPFA, the Fourier transform was estimated [4]. Some threshold curves have been studied [5], but they are derived for additive noise models. Since we have a Poisson noise model, we have used the more conservative threshold of 0.5 for our calculations. Ten images were used to construct each cell of the tables in the form of the mean of the metric plus or minus its standard deviation.

The PSNR dies off much quicker than the FRC values for the same image. For the $100 \%$ and $80 \%$ sampled versions of the truth image, the resolution only dies off when the dose is 5. However, the PSNR dies off rapidly as the dose is reduced. For the 1000, 500, and 50 dose images, the FRC is the maximum, or close, until we undersample at $20 \%$. The PSNR for these values tapers down as we get into the bottom right hand corner of the table, even though the resolution remains high. Overall, we find that undersampled images can be reconstructed to acceptable resolution even when the dose per pixel is also reduced [6]. 
References:

[1] A Stevens, H Yang, L Carin et al. Microscopy 63(1), (2014), pp. 41.

[2] A Stevens, L Kovarik, P Abellan et al. Advanced Structural and Chemical Imaging 1(1), (2015), pp. 1.

[3] M Zhou, H Chen, J Paisley et al. Image Processing, IEEE Transactions on 21(1), (2012), pp. 130.

[4] V. Y. Liepin'sh. Automatic control and computer sciences 30(3), (1996), pp. 20.

[5] M. Van Heel and M. Schatz. Journal of structural biology 151(3), (2005), pp. 250.

[6] Supported by the Chemical Imaging, Signature Discovery, and Analytics in Motion initiatives at PNNL. PNNL is operated by Battelle Memorial Inst. for the US DOE; contract DE-AC05-76RL01830.

\begin{tabular}{c|cccc|cccc} 
Pixels & \multicolumn{4}{|c}{ Without Reconstruction } & \multicolumn{4}{c}{ With Reconstruction } \\
Dose & $100 \%$ & $80 \%$ & $40 \%$ & $20 \%$ & $100 \%$ & $80 \%$ & $40 \%$ & $20 \%$ \\
\hline 1000 & $\mathbf{3 . 9} \pm \mathbf{0}$ & $\mathbf{3 . 9} \pm \mathbf{0}$ & $\mathbf{2 . 2} \pm \mathbf{0}$ & $1.2 \pm 0.1$ & $\mathbf{3 . 9} \pm \mathbf{0}$ & $\mathbf{3 . 9} \pm \mathbf{0}$ & $\mathbf{3 . 9} \pm \mathbf{0}$ & $\mathbf{3 . 9} \pm \mathbf{0}$ \\
500 & $\mathbf{3 . 9} \pm \mathbf{0}$ & $\mathbf{3 . 6} \pm \mathbf{0 . 2}$ & $\mathbf{2 . 1} \pm \mathbf{0}$ & $1.2 \pm 0$ & $\mathbf{3 . 9} \pm \mathbf{0}$ & $\mathbf{3 . 9} \pm \mathbf{0}$ & $\mathbf{3 . 9} \pm \mathbf{0}$ & $\mathbf{3 . 9} \pm \mathbf{0}$ \\
50 & $\mathbf{2 . 3} \pm \mathbf{0 . 1}$ & $\mathbf{2 . 1} \pm \mathbf{0}$ & $\mathbf{1 . 9} \pm \mathbf{0 . 1}$ & $1.0 \pm 0.2$ & $\mathbf{3 . 9} \pm \mathbf{0}$ & $\mathbf{3 . 9} \pm \mathbf{0}$ & $\mathbf{3 . 9} \pm \mathbf{0}$ & $\mathbf{3 . 6} \pm \mathbf{0 . 3}$ \\
5 & $\mathbf{1 . 4} \pm \mathbf{0 . 2}$ & $1.2 \pm 0.1$ & $0.7 \pm 0$ & $0.6 \pm 0.1$ & $\mathbf{3 . 7} \pm \mathbf{0 . 4}$ & $\mathbf{3 . 2} \pm \mathbf{0 . 3}$ & $1.2 \pm 0$ & $1.1 \pm 0.1$ \\
2.5 & $1.0 \pm 0.2$ & $0.8 \pm 0$ & $0.6 \pm 0.1$ & $0.4 \pm 0$ & $1.2 \pm 0$ & $1.2 \pm 0.1$ & $1.0 \pm 0$ & $0.8 \pm 0.2$
\end{tabular}

Table 1: The FRC resolutions $\left(\AA^{-1}\right)$. The bold entries indicate atomic resolution $\left(1.22 \AA^{-1}\right)$.

\begin{tabular}{c|cccc|cccc} 
Pixels & \multicolumn{4}{|c}{ Without Reconstruction } & \multicolumn{4}{c}{ With Reconstruction } \\
Dose & $100 \%$ & $80 \%$ & $40 \%$ & $20 \%$ & $100 \%$ & $80 \%$ & $40 \%$ & $20 \%$ \\
\hline 1000 & $\mathbf{3 0 . 7} \pm \mathbf{1 . 0}$ & $\mathbf{1 4 . 9} \pm \mathbf{0}$ & $\mathbf{1 0 . 3} \pm \mathbf{0}$ & $9.0 \pm 0$ & $\mathbf{3 1 . 3} \pm \mathbf{1 . 3}$ & $\mathbf{3 1 . 5} \pm \mathbf{1 . 2}$ & $\mathbf{3 1 . 4} \pm \mathbf{1 . 2}$ & $\mathbf{2 5 . 7} \pm \mathbf{0 . 5}$ \\
500 & $\mathbf{2 7 . 4} \pm \mathbf{0 . 6}$ & $\mathbf{1 4 . 9} \pm \mathbf{0}$ & $\mathbf{1 0 . 2} \pm \mathbf{0}$ & $9.0 \pm 0$ & $\mathbf{2 8 . 3} \pm \mathbf{0 . 8}$ & $\mathbf{2 8 . 6} \pm \mathbf{0 . 8}$ & $\mathbf{2 8 . 0} \pm \mathbf{0 . 8}$ & $\mathbf{2 4 . 6} \pm \mathbf{0 . 7}$ \\
50 & $\mathbf{1 7 . 5} \pm \mathbf{0 . 4}$ & $\mathbf{1 3 . 4} \pm \mathbf{0 . 1}$ & $\mathbf{1 0 . 0} \pm \mathbf{0}$ & $8.9 \pm 0$ & $\mathbf{1 8 . 2} \pm \mathbf{0 . 5}$ & $\mathbf{1 8 . 3} \pm \mathbf{0 . 5}$ & $\mathbf{1 8 . 7} \pm \mathbf{0 . 8}$ & $\mathbf{1 7 . 5} \pm \mathbf{0 . 7}$ \\
5 & $\mathbf{1 1 . 5} \pm \mathbf{0 . 1}$ & $10.6 \pm 0$ & $9.2 \pm 0$ & $8.6 \pm 0$ & $\mathbf{1 2 . 8} \pm \mathbf{0 . 2}$ & $\mathbf{1 2 . 8} \pm \mathbf{0}$ & $12.9 \pm 0.4$ & $12.6 \pm 0.5$ \\
2.5 & $10.3 \pm 0.2$ & $9.7 \pm 0.1$ & $8.8 \pm 0$ & $8.4 \pm 0$ & $12.3 \pm 0.4$ & $12.3 \pm 0.4$ & $12.3 \pm 0.4$ & $12.4 \pm 0.4$
\end{tabular}

Table 2: The PSNR values $(\mathrm{dB})$. The bold entries indicate atomic resolution $\left(1.22 \AA^{-1}\right)$.

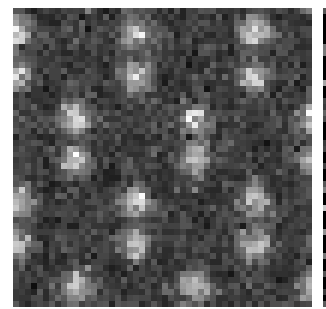

(a)

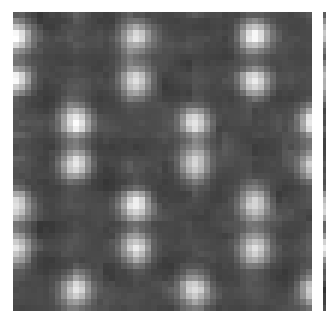

(d)

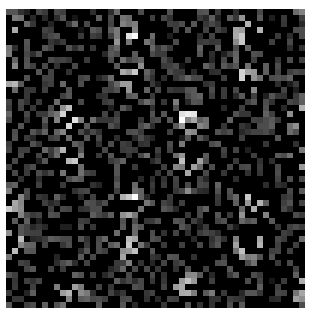

(b)

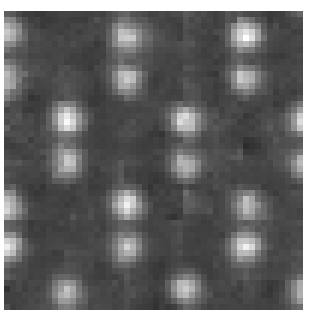

(e)

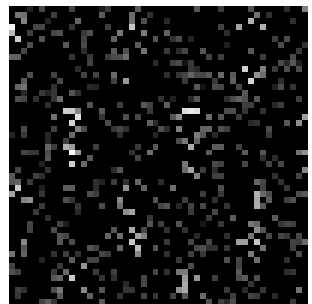

(c)

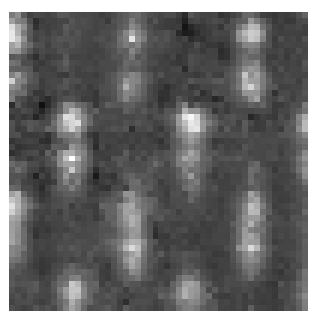

(f)

Figure 1: Some of the BPFA reconstructions used to populate Tables 1 and 2. All of these images had Poisson noise applied to simulate a dose of 50. Figures (a), (b), and (c) are the noisy images with sampling percentages of $100 \%, 40 \%$, and 20\%, respectively. Figures (d), (e), and (f) are their reconstructions. 